\title{
Conformable Kesirsel Mertebeden Tam Değer Fonksiyonlu Lojistik Modelin Kararlılık ve Çatallanma Analizi Güven KAYA ${ }^{1 *}$, Şenol KARTAL ${ }^{2}$
}

\author{
${ }^{1}$ Bingöl Üniversitesi, Fen Edebiyat Fakültesi, Matematik Bölümü, Bingöl \\ ${ }^{2}$ Nevşehir Hacı Bektaş Veli Üniversitesi, Eğitim Fakültesi, Matematik ve Fen Bilimleri Eğitimi Bölümü, Nevşehir \\ (ORCID:0000-0003-0411-5633) (ORCID: 0000-0003-1205-069X)
}

$\ddot{O} \mathbf{z}$

Bu çalışmada, conformable kesirsel mertebeden tam değer fonksiyonlu lojistik model ele alınmıștır. Modele tam değer fonksiyonlarının kullanılmasına dayalı bir ayrıklaştırma işlemi uygulanılarak bir fark denklem sistemi elde edilmiştir. Elde edilen bu fark denklem sisteminin pozitif denge noktasının yerel asimptotik kararlı olmasını sağlayan cebirsel koşullar Schur-Cohn kriterlerinin kullanılmasıyla elde edilmiştir. Yine çatallanma analizi ile sistemde $r$ parametresinin değişimine bağlı olarak Neimark-Sacker çatallanmasının oluştuğu gösterilmiştir. Ayrıca kesirsel mertebeden türev parametresi $(\alpha)$ ve kesiklileştirme parametresi $(h)$ nin sistemin dinamik yapısı üzerindeki etkisi araştırılmıştır. Elde edilen tüm teorik sonuçlar nümerik simülasyonlarla desteklenmiştir.

Anahtar kelimeler: Conformable kesirli mertebeden türev, tam değer fonksiyonu, kararll1ık, Neimark-Sacker çatallanma.

\section{Stability and Bifurcation Analysis of a Conformable Fractional Order Logistic Model with Piecewise Constant Arguments}

\begin{abstract}
In this study, a conformable fractional order logistic model with piecewise constant arguments is considered. A discrete system is obtained by applying a discretization process to the model based on the use of piecewise constant arguments. By using the Schur-Cohn criterion, necessary and sufficient condition is obtained for asymptotic stability of a positive equilibrium point of the discrete system. Moreover, bifurcation analysis shows that NeimarkSacker bifurcation occurs due to the change of parameter $r$ in the system. In addition, effect of fractional order parameter $(\alpha)$ and discretization parameter $(h)$ on the dynamic structure of the system are investigated. Finally, all theoretical results are supported by numerical simulations.
\end{abstract}

Keywords: Conformable fractional derivative, Piecewise constant arguments, Stability, Neimark-Sacker bifurcation.

\section{Giriş}

Kesirli analiz, klasik diferansiyelin ve integrasyonun keyfi mertebeye (tamsayı olmayan duruma) genelleştirilmesidir. Kesirli integral ve türevin; Riemann-Liouville kesirli integrasyon ve türev, Caputo kesirli türev, Grünwald-Letnikov kesirli türev gibi birçok tanımı vardır.

$\alpha \in(0,1)$ mertebeli Riemann-Liouville kesirli türevi

$$
\frac{d^{\alpha}}{d t^{\alpha}} f(t)=\frac{1}{\Gamma(1-\alpha)} \frac{d}{d t} \int_{0}^{t}(t-s)^{-\alpha} f(s) d s
$$

şeklinde tanımlanmıştır [1]. Standart başlangıç koşullarına sahip kesirli başlangıç değer problemlerinin olası çözümlerini elde etmek amacıyla Riemann-Liouville kesirli türevi yeniden yorumlanarak

"Sorumlu yazar: gkaya@bingol.edu.tr

Geliş Tarihi: 26.12.2019, Kabul Tarihi: 04.06.2020 


$$
D^{\alpha} f(t)=\frac{1}{\Gamma(1-\alpha)} \int_{0}^{t} \frac{f^{(m)}(\tau)}{(t-\tau)^{\alpha+1-m}} d \tau
$$

şeklinde Caputo kesirli türev tanımı türetilmiştir [1]. Bu nedenle günümüzde uygulamalı bilimciler tarafindan en çok rağbet edilen tanım Caputo kesirli türevidir [2].

2014 yılında Khalil ve arkadaşları [3] tarafından "conformable kesirli türev" adı altında yeni bir kesirli türev tanımı ortaya atılmıştır. Daha sonra 2015 de Abdeljawad [4] tarafindan sol/sağ conformable kesirli türev tanımlanmıştır. Bu tanıma göre $\alpha \in(0,1]$ olmak üzere f: $[a, \infty) \rightarrow$ IR fonksiyonunun sol conformable kesirli türevi

$$
\left({ }_{\alpha}^{a} T f\right)(t)=\lim _{\varepsilon \rightarrow 0} \frac{f\left(t+\varepsilon(t-a)^{1-\alpha}\right)-f(t)}{\varepsilon},
$$

şeklinde ve sağ conformable kesirli türev ise

$$
\left({ }_{\alpha}^{b} T f\right)(t)=-\lim _{\varepsilon \rightarrow 0} \frac{f\left(t+\varepsilon(b-t)^{1-\alpha}\right)-f(t)}{\varepsilon},
$$

olarak tanımlanır. Dikkat edelim ki eğer $f$ diferansiyellenebilir ise o halde

$$
\left({ }_{\alpha}^{a} T f\right)(t)=(t-a)^{1-\alpha} f^{\prime}(t) \text { ve }\left({ }_{\alpha}^{b} T f\right)(t)=(b-t)^{1-\alpha} f^{\prime}(t)
$$

dir. Ayrıca literatürde bu türevin pekçok biyolojik ve fiziksel uygulamaları bulunmaktadır [5-8].

Son dönemlerde kesirli mertebeli diferansiyel denklemler araştırmacıların dikkatini çekmektedir. Birçok araştırmacı kesirli mertebeli adi diferansiyel denklemler ile oluşturulmuş matematiksel modellerin klasik tamsayı mertebeli diferansiyel denklemlerle oluşturulan modellere göre daha başarılı sonuçlar verdiğini göstermiştir [8-19]. Kesirli mertebeli diferansiyel denklemlerin kullanılmasında iki ana neden vardır. Bunlar birçok biyolojik olayda var olan sistem hafiza etkisi ve kalıtsal özelliklerinin bu denklemler vasıtası ile tanımlanabiliyor olmasıdır. Bugün kesirli mertebeli diferansiyel denklemler akışkanlar dinamiği, mekanik, biyoloji, fizik, epidemoloji ve mühendislik gibi birçok alanda uygulamaya sahiptir [20-26]. Araştırmacılar tüm fiziksel olayların kesirli mertebeli diferansiyel denklemlerden oluştuğunu ve kesirli mertebeli diferansiyel denklemlerin tamsayı mertebeli diferansiyel denklemlerin genelleştirilmiş hali olduğunu göstermişlerdir [26].

Lineer olmayan kesirli mertebeli diferansiyel denklemlerin birçoğu analitik çözüme sahip değildir. Bu nedenle Adomain decomposition metot [27], homotopi perturbasyon metot [28], homotopi analiz metot [29], varyasyonel iterasyon metot [30], Adams Type predictor-corrector metot [31], Piecewise Constant Argument metot [21] ve diferansiyel transform metot [32] gibi nümerik metotlar kesirli mertebeli diferansiyel denklemlerin çözümünde kullanılmıştır.

Popülasyon dinamiğinde en temel modellerden birisi lojistik modeldir ve 1838 y1lında Verhulst [33] tarafından oluşturulmuştur. Bu modelin Caputo anlamda kesirsel mertebeden versiyonu

$$
D^{\alpha} N(t)=\mathrm{rN}(\mathrm{t})\left(1-\frac{\mathrm{N}(t)}{\mathrm{K}}\right)
$$

şeklinde ifade edilir. Burada $\mathrm{r}$ birim popülasyon büyüme oranını ve $K$ taşıma kapasitesini temsil etmektedir. $u(t)=\frac{\mathrm{N}(t)}{\mathrm{K}}$ dönüşümü altında (2) modeli

$$
D^{\alpha} u(t)=r u(t)(1-u(t))
$$

formuna indirgenir. Literatürde (3) modelinin çözümlerinin varlığı ve tekliğí, denge noktalarının kararlılığ1 ve çeşitli biyolojik uygulamaları mevcuttur [1, 34-36]

El-Raheem ve arkadaşları [21], Caputo kesirsel mertebeden türeve sahip (3) lojistik denklemine tam değer fonksiyonu ekleyerek 


$$
D^{\alpha} x(t)=r x\left(\left[\frac{t}{h}\right] h\right)\left(1-x\left(\left[\frac{t}{h}\right] h\right)\right)
$$

modelini göz önüne almışlardır. (4) denkleminin $t \in[n h,(n+1) h)$ alt aralıklarında çözümünden ise

$$
x_{n+1}=x_{n}+\frac{h^{\alpha}}{\Gamma(1+\alpha)} r x_{n}\left(1-x_{n-1}\right)
$$

fark denklemini elde etmişlerdir.

$\mathrm{Bu}$ çalışmanın amacı ise aşağıdaki gibi verilmiş olan tam değer fonksiyonlu conformable kesirli mertebeden lojistik modelin dinamik davranışlarını incelemektir.

$$
\mathrm{T}_{\alpha} \mathrm{x}(\mathrm{t})=\operatorname{rx}(\mathrm{t})\left(1-\frac{\mathrm{x}\left(\left[\frac{\mathrm{t}-\mathrm{h}}{\mathrm{h}}\right] \mathrm{h}\right)}{\mathrm{k}}\right)
$$

burada $x(\mathrm{t})$ popülasyon yoğunluğunu, $\mathrm{r}$ popülasyon büyüme oranını, $k$ taşıma kapasitesini, $h$ kesiklileştirme parametresini temsil etmektedir ve parametrelerin tamamı pozitiftir. $[\mathrm{t}], \mathrm{t} \in(0, \infty)$ için $\mathrm{t}$ nin tam değerini göstermektedir.

\section{Fark Denklem Sistemine Geçiş}

Bu kısımda Kartal ve Gürcan'ın [7] çalışmasında ortaya koydukları kesiklileştirme yöntemi kullanilacaktır.

Sol conformable türevin (1) özelliğinden (6) denklemi $t \in[n h,(n+1) h), n=0,1,2, \ldots$ alt aralığında göz önüne alındı̆̆ında

$$
(t-n h)^{1-\alpha} \frac{d x(t)}{d t}=r x(t)\left(1-\frac{x(n h-h)}{k}\right)
$$

şeklinde yazılabilir. Bu denklemin yeniden düzenlenmesiyle

$$
\frac{d x(t)}{x(t)}=r\left(1-\frac{x(n h-h)}{k}\right)(t-n h)^{\alpha-1} d t
$$

şeklinde değişkenlerine ayrılabilir bir diferansiyel denklem elde edilebilir. (8) denkleminin [nh,t) aralığında t ye göre integrali alındığında

$$
x(t)=x(n h) e^{r\left(1-\frac{x(n h-h)}{k}\right) \frac{(t-n h)^{\alpha}}{\alpha}}
$$

bulunur. Bu ifadede $t \rightarrow(n+1) h$ olarak alınırsa ve $x(n h)$ ile $y(n h)$ sırasılyla $x(n)$ ve $y(n)$ şeklinde değiştirildiğinde,

$$
x_{n+1}=x(n) e^{r\left(1-\frac{x(n-1)}{k}\right) \frac{h^{\alpha}}{\alpha}}
$$

elde edilir. $u_{1}(n)=x(n)$ ve $u_{2}(n)=x(n-1)$ değişken değiştirmesi ile

$$
\left\{\begin{array}{l}
u_{1}(n+1)=u_{1}(n) e^{r\left(1-\frac{u_{2}(n)}{k}\right) \frac{h^{\alpha}}{\alpha}} \\
u_{2}(n+1)=u_{1}(n)
\end{array}\right.
$$

şeklinde bir fark denklem sistemi elde edilir. 


\section{Yerel Asimptotik Kararlılık Analizi}

Bu kısımda fark denklemleri için denge noktası tanımı verilecek ve (11) fark denklem sisteminin pozitif denge noktasının yerel asimptotik kararlı olmasını sağlayan cebirsel koşullar Schur-Cohn kriterlerinin kullanılmasıyla elde edilmeye çalışılacaktır.

Tanım 1: $x_{n}=x_{n+1}=x^{*}$ eşitliğini sağlayan $x^{*}$ noktasına fark denkleminin denge noktası denir [37]. Bu tanım dikkate alındığında $u_{1}(n)=u_{1}(n+1)=x^{*}$ ve $u_{2}(n)=u_{2}(n+1)=y^{*}$ olmak üzere $(11)$ fark denklem sisteminin ikinci denkleminden $x^{*}=y^{*}$ olur. Bu ifade sistem (11) in birinci denkleminde yerine yazıl111r ise

$x^{*}=x^{*} e^{r\left(1-\frac{x^{*}}{k}\right) \frac{h^{\alpha}}{\alpha}}$,

elde edilir. Buradan $x^{*}=0$ veya $x^{*}=k$ olarak bulunur. Öyle ise sistem (11) in pozitif denge noktas1 $E^{*}=(k, k)$ dir.

Teorem 1: (Schur-Chon Kriteri ). $\lambda_{i}, x(n+1)=A x(n)$ fark denklem sistemindeki $2 x 2$ boyutlu $A$ matrisinin bütün öz değerleri ve

$$
\lambda^{2}+p_{1} \lambda+p_{0}=0
$$

da $A$ matrisinin karakteristik polinomu olsun. Bu taktirde (12) denkleminin bütün kökleri birim dairenin içindedir $\left(\left|\lambda_{i}\right|<1\right)$ ancak ve ancak

(a) $1+p_{1}+p_{0}>0$,

(b) $1-p_{1}+p_{0}>0$,

(c) $1-p_{0}>0$.

(11) sistemin $E^{*}=(k, k)$ denge noktasındaki Jakobyen matrisi

$J_{E^{*}}=\left(\begin{array}{cc}1 & -\frac{h^{\alpha} r}{\alpha} \\ 1 & 0\end{array}\right)$

ve Jakobyen matrise karşılık gelen karakteristik denklem ise

$\lambda^{2}-\lambda+\frac{h^{\alpha} r}{\alpha}=0$

dir. Burada $\mathrm{p}_{1}=-1$ ve $\mathrm{p}_{0}=\frac{h^{\alpha} r}{\alpha}$ dır. Schur-Cohn kriterlerinin (a) şartından $1+p_{1}+p_{0}=\frac{\mathrm{h}^{\alpha} \mathrm{r}}{\alpha}>0$;

(b) şartından $1-p_{1}+p_{0}=2+\frac{\mathrm{h}^{\alpha} \mathrm{r}}{\alpha}>0$ elde edilir. (c) şartından $1-p_{0}=1-\frac{\mathrm{h}^{\alpha} \mathrm{r}}{\alpha}>0$ ve buradan $r<\alpha h^{-\alpha}$ elde edilir.

$\mathrm{Bu}$ sonuçlar altında aşağıdaki teoremi verebiliriz.

Teorem 2: Sistem (11) in pozitif denge noktası $E^{*}=(k, k)$ nın lokal asimptotik kararlı olması için gerek ve yeter şart $r<r^{*}=\alpha h^{-\alpha}$ olmasıdır. 


\section{Neimark-Sacker Çatallanma Analizi}

Neimark-Sacker çatallanması, kesikli zamanlı dinamik sistemlerde ortaya çıkan bir çatallanma türüdür. $\mathrm{Bu}$ çatallanma sonucunda bir limit döngüsünün oluşması beklenir. (11) fark denklem sisteminde Neimark-Sacker çatallanma analizi için $r$ çatallanma parametresi olarak seçilecektir.

Teorem 3: Eğer $r=r^{*}$ ve $q \neq 0$ ise bu durumda (11) sistemi için $E^{*}$ pozitif denge noktası civarında Neimark-Sacker çatallanması oluşur. Üstelik $q<0$ ise bu durumda oluşan çatallanma supercritical Neimark-Sacker çatallanması, $q>0$ ise subcritical Neimark-Sacker çatallanmasıdır.

İspat: $1-p_{0}=0$ eşitliğinden Neimark-Sacker çatallanma değeri $r^{*}=\alpha h^{-\alpha}$ şeklinde elde edilir. $r=r^{*}$ olmak üzere (11) fark denklem sisteminin pozitif denge noktasındaki lineerleştirilmiş sisteminin $J_{E^{*}}\left(r^{*}\right)$ Jakobiyen matrisine karşıllk gelen karakteristik denklemi,

$$
\lambda^{2}-\lambda+1=0
$$

biçimindedir. Buradan $\lambda$ öz değerleri

$\lambda_{1,2}\left(r^{*}\right)=\frac{1}{2} \pm i \frac{\sqrt{3}}{2}=a \pm i b$,

şeklinde elde edilir. Buradan kolayca görülebilir ki $\left|\lambda_{1,2}\left(r^{*}\right)\right|=1$ dir. Diğer taraftan Transversality koşulundan

$\left.\frac{d\left|\lambda_{1,2}(r)\right|}{d r}\right|_{r=r^{*}}=i \frac{h^{\alpha}}{\alpha \sqrt{2}} \neq 0$,

olduğu görülür. $-p_{1} \neq 0,-1$ Nonresonance koşulundan $\lambda_{1,2}^{n}\left(r^{*}\right) \neq 1, n=1,2,3,4$ bulunur. Sonuç olarak sistem (11) için Theorem (2) de verilen şartlar altında Neimark-Sacker çatallanması ortaya çıkar. Şimdi çatallanmanın yönünü belirlemek için q değerini bulalım. Bunun için ilk olarak denge noktasını orjine taşıyalım.

$u=u_{1}-u_{1}^{*}$ ve $v=u_{2}-u_{2}^{*}$ dönüşümleri altında (11) sistemini

$$
\left(\begin{array}{l}
u \\
v
\end{array}\right) \rightarrow\left(\begin{array}{cc}
1 & -1 \\
1 & 0
\end{array}\right)\left(\begin{array}{l}
u \\
v
\end{array}\right)+\left(\begin{array}{c}
f(u, v) \\
0
\end{array}\right)
$$

şeklinde yazabiliriz. Burada

$$
f(u, v)=-\frac{1}{k} u v+\frac{1}{2 k} v^{2}+\frac{1}{2 k^{2}} u v^{2}-\frac{1}{6 k^{2}} v^{3}+o\left((|u|+|v|)^{4}\right),
$$

dir.

$T=\left(\begin{array}{cc}\frac{\sqrt{3}}{2} & \frac{1}{2} \\ 0 & 1\end{array}\right)$

olmak üzere $\left(\begin{array}{l}u \\ v\end{array}\right)=T\left(\begin{array}{l}X \\ Y\end{array}\right)$ dönüşümünü göz önüne alalım. O halde (14) dönüşümü

$$
f\left(\begin{array}{l}
X \\
Y
\end{array}\right)=\left(\begin{array}{cc}
a & -b \\
b & a
\end{array}\right)\left(\begin{array}{l}
X \\
Y
\end{array}\right)+\left(\begin{array}{l}
F_{1}(X, Y) \\
F_{2}(X, Y)
\end{array}\right)
$$

şeklini alır ve burada 
$F_{1}(X, Y)=-\frac{X Y}{k}+\frac{X Y^{2}}{2 k^{2}}+\frac{Y^{3}}{6 \sqrt{3} k^{2}}+o\left((|X|+|Y|)^{4}\right)$

$\mathrm{Ve}$

$F_{2}(X, Y)=0$

dir. Buradan

$\xi_{20}=\frac{1}{8}\left(\left(F_{1 X X}-F_{1 Y Y}+2 F_{2 X Y}\right)+i\left(F_{2 X X}-F_{2 Y Y}-2 F_{1 X Y}\right)\right)=\frac{i}{4 k}$,

$\xi_{11}=\frac{1}{4}\left(\left(F_{1 X X}+F_{1 Y Y}\right)+i\left(F_{2 X X}+F_{2 Y Y}\right)\right)=0$,

$\xi_{02}=\frac{1}{8}\left(\left(F_{1 X X}-F_{1 Y Y}-2 F_{2 X Y}\right)+i\left(F_{2 X X}-F_{2 Y Y}+2 F_{1 X Y}\right)\right)=-\frac{i}{4 k}$

ve

$\xi_{21}=\frac{1}{16}\left(\left(F_{1 X X X}+F_{1 X Y Y}+F_{2 X X Y}+F_{2 Y Y Y}\right)+i\left(F_{2 X X X}+F_{2 X Y Y}-F_{1 X X Y}-F_{1 Y Y Y}\right)\right)=\frac{1}{16 k^{2}}-\frac{i \sqrt{3}}{48 k^{2}}$ olarak elde edilir. Sonuç olarak q katsayısı

$q=-\operatorname{Re}\left[\frac{(1-2 \lambda) \bar{\lambda}^{2}}{1-\lambda} \xi_{11} \xi_{20}\right]-\frac{1}{2}\left|\xi_{11}\right|^{2}-\left|\xi_{02}\right|^{2}+\operatorname{Re}\left(\bar{\lambda} \xi_{21}\right)=-\frac{1}{16 k^{2}}<0$

şeklinde hesaplanır. Bu son ifadeden (11) fark denklem sistemde oluşan Neimark-Sacker çatallanmasının supercritical Neimark-Sacker çatallanması olduğu söylenebilir.

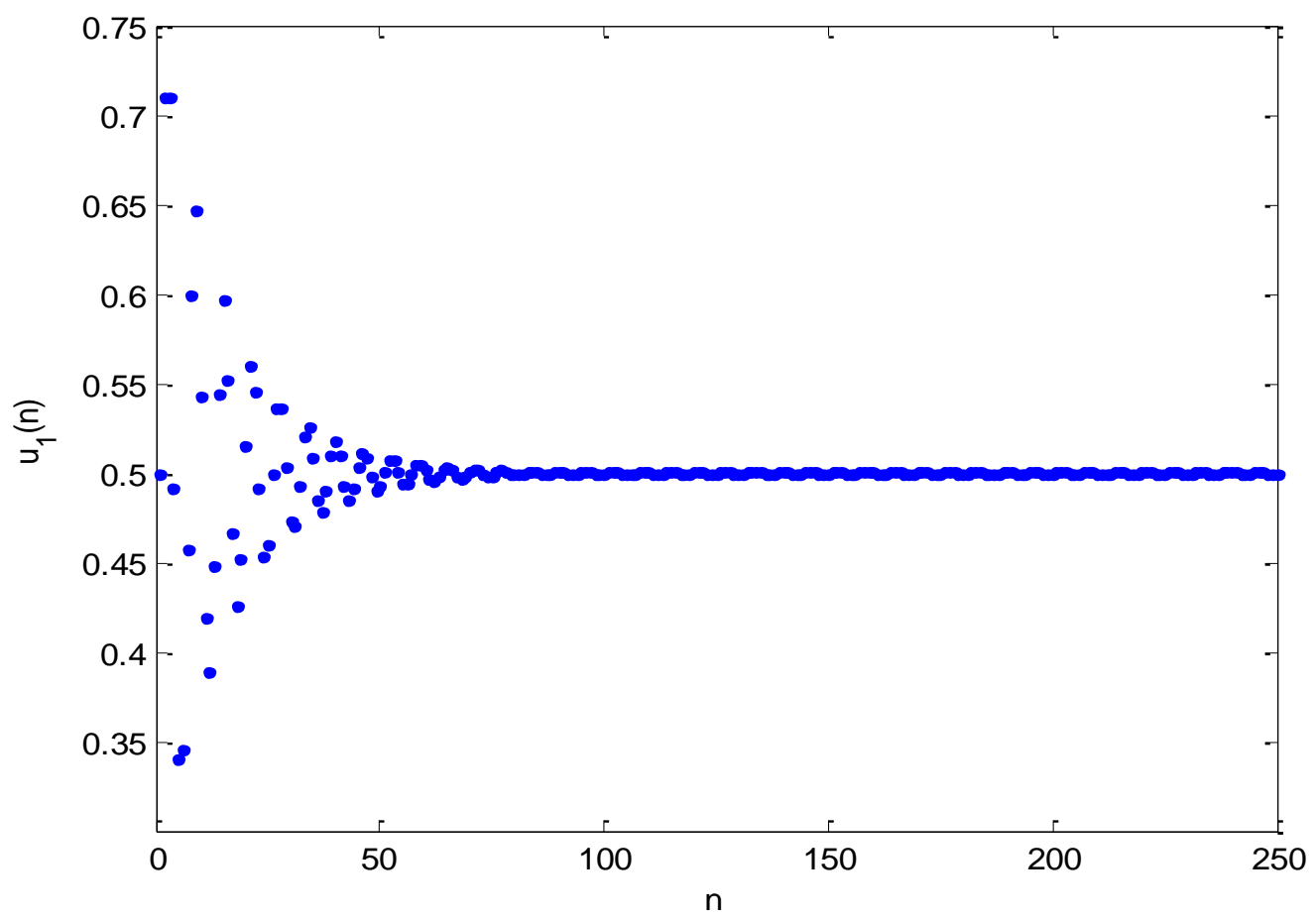

Şekil 1. $\alpha=0.5, h=0.95, r=0.45$ ve $k=0.5$ parametre değerleri için (11) fark denklem sisteminin çözüm dizisinin grafiği 

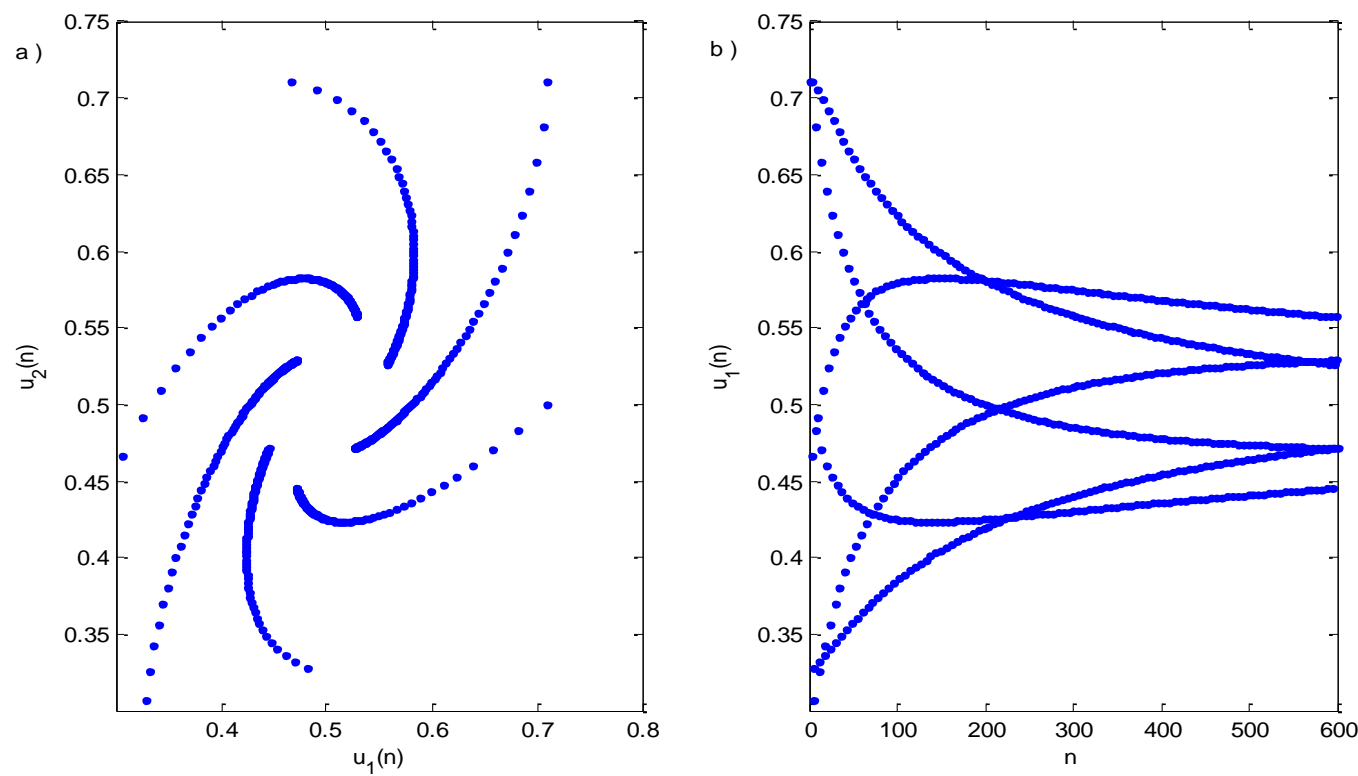

Şekil 2. $r=0.52989$ için Supercritical Neimark-Sacker çatallanması (a); time series grafiği (b). Diğer parametre değerleri Şekil 1'deki gibidir.

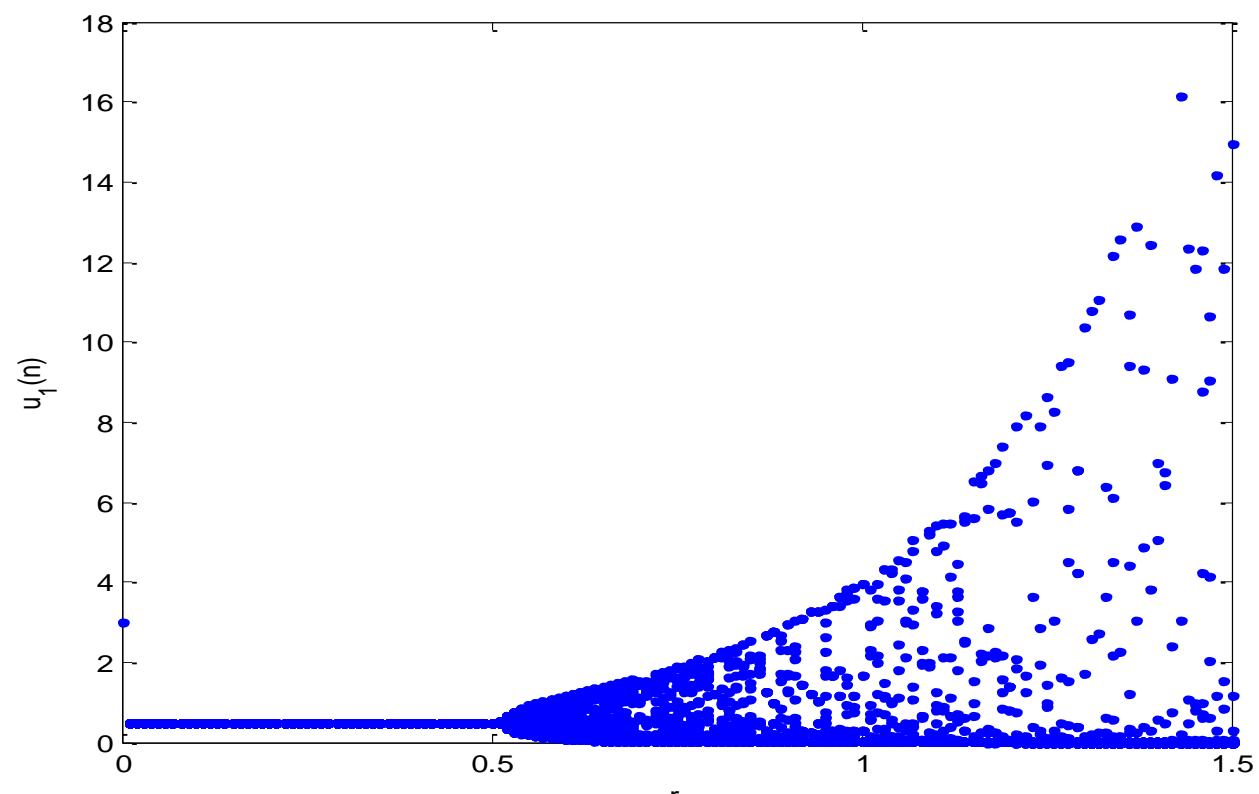

Şekil 3. Şekil 1'deki parametre değerleri için r ye karşı çizilmiş Neimark-Sacker çatallanma grafiği. 

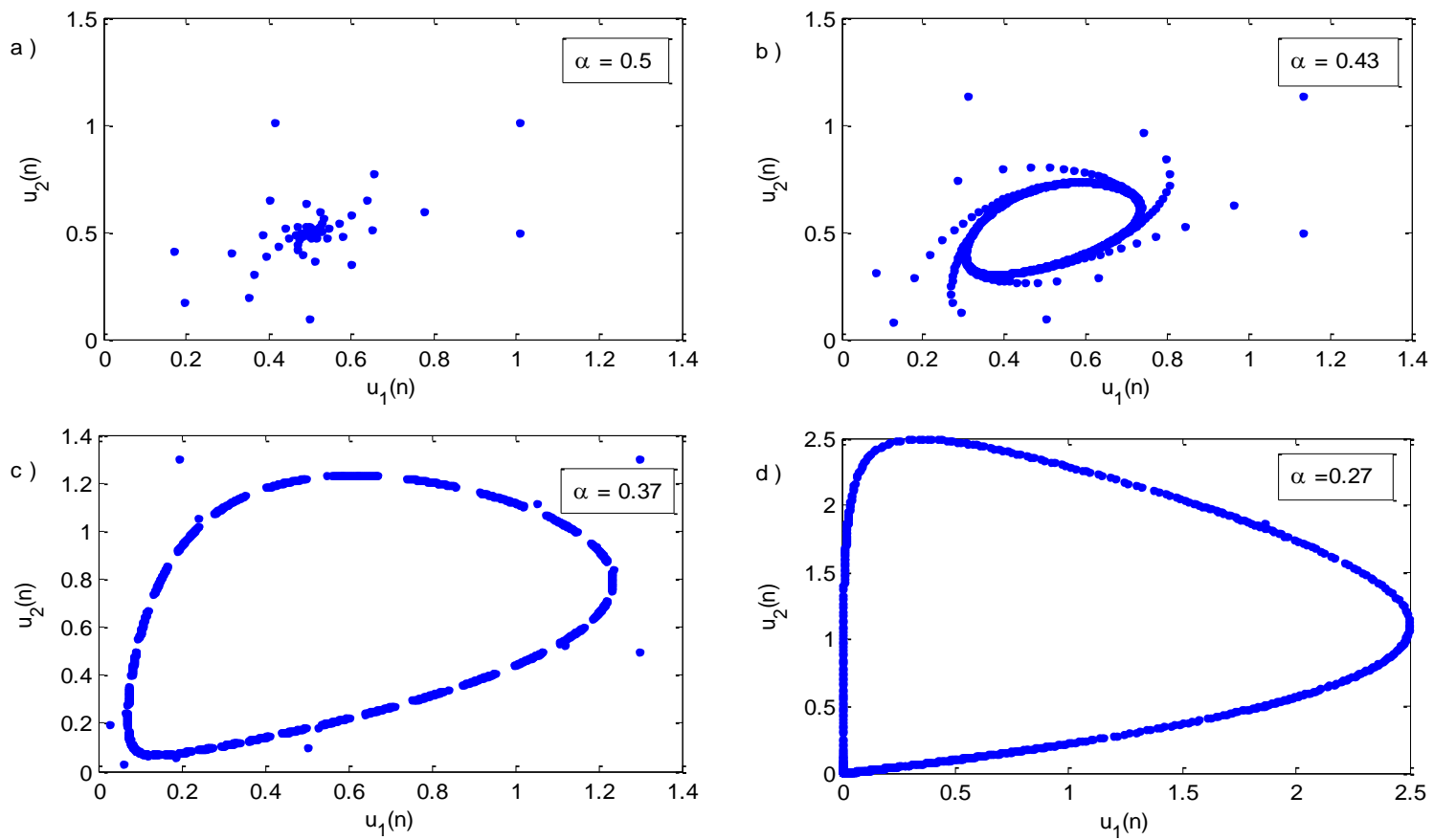

Şekil 4. $\mathrm{h}=0.95, \mathrm{k}=0.5, r=0.45$ olmak üzere $\alpha$ nın farklı değerleri için çizilmiş faz diyagramı
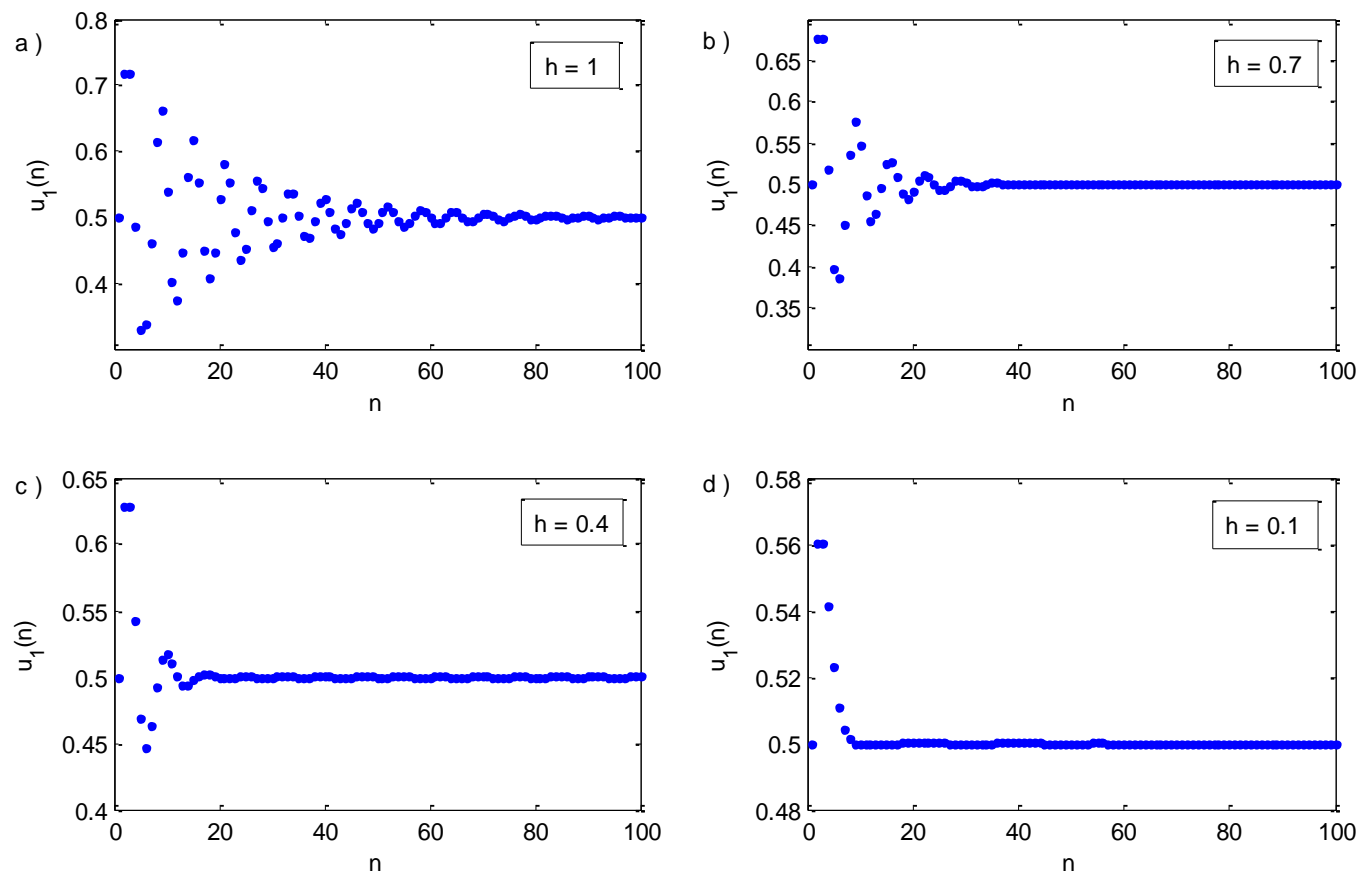

Şekil 5. $r=0.45, k=0.5$ ve $\alpha=0.5$ olmak üzere $h$ nın farklı değerleri için çizilmiş time series grafikleri.

\section{Sonuç}

$\mathrm{Bu}$ çalışmada conformable kesirsel mertebeden tam değer fonksiyonlu lojistik model ele alınmıştır. Modelin $[n h,(n+1) h)$ alt aralığındaki çözümünden (11) fark denklem sistemi elde edilmiştir. (11) fark denklem sisteminin $E^{*}=(k, k)$ pozitif denge noktasının yerel asimptotik kararlı olmasını sağlayan cebirsel koşul $r<\alpha h^{-\alpha}$ olarak bulunmuştur. $\alpha=0.5, h=0.95$ için bu koşuldan yerel asimptotik kararlılık aralığı nümerik olarak $r<0.512989$ olarak belirlenmiştir. Şekil 1'den $r$ bu aralık içinde kalacak şekilde seçildiğinde $(r=0.45)$ sistemin pozitif denge noktasının yerel asimptotik kararlı 
olduğu görülebilir. Neimark-Sacker çatallanma analizi ile kritik çatallanma değerinin $r^{*}=\alpha h^{-\alpha}$ olduğu gösterilmiştir ve oluşan çatallanmanın supercritical çatallanma olduğu ispatlanmıştır $\left(q=-\frac{1}{16 \mathrm{k}^{2}}<0\right)$. $\alpha=0.5, h=0.95$ için kritik çatallanma değeri $r^{*}=0.512989$ olarak hesaplanmıştır ( Şekil 2 ve Şekil 3 ). Son olarak Şekil 4 ve Şekil 5 den kesirsel mertebeden türev parametresi $(\alpha)$ ve kesiklileştirme parametresi $(h)$ nin sistemin dinamik yapısındaki etkisi görülebilir.

\section{5. Öneriler}

Son yıllarda kesirsel mertebeden dinamik sistemlerin araştırılmasında çok büyük bir artış gözlenmektedir. Biyoloji, Fizik, Kimya ve Mühendislik gibi alanlarda bu denklemler yardımıyla pek çok biyolojik ve fiziksel olay başarılı bir şekilde modellenmiştir. Özellikle sistemlerde var olan hafiza etkisi göz önüne alındığında bu tür dinamik sistemlerin araştırılmasının ne kadar önemli olduğu ortaya çıkmaktadır. Ancak Riemann-Liouville ve Caputo gibi kesirsel mertebeden türevler bir integral denklemi ile tanımlanmaktadır ve bu integral denklemlerin çekirdekleri lokal olmayıp bir singularitiye sahiptir. Dahası bu türevler vasıtası ile oluşturulmuş diferansiyel denklemlerin çoğunun analitik çözümünü bulmak imkansız hale gelmektedir. $\mathrm{Bu}$ nedenle çeşitli nümerik yöntemlere ihtiyaç duyulmaktadır. Ayrıca bu türevlerin geometrik yorumları da hala tam olarak bilinmemektedir. Bu gibi zorluklar akla şu soruyu getirmektedir: Ancak tam sayı mertebeden türev tanımının bir uzantısı olarak yeni bir kesirsel mertebeden türev tanımlanabilir mi? Son yıllarda bu sorunun cevabı olarak birçok yeni kesirsel mertebeden türev tanımı ortaya atılmıştır. Conformable kesirsel mertebeden türev de bu yeni türev tanımlarından birisidir. Literatürde conformable kesirsel mertebeden diferansiyel denklemlerin çözümlerinin varlığı-tekliği, çözüm yöntemleri, vs. ile ilgili birçok çalışma yapılmış olmasına rağmen bu tür denklemlerin popülasyon dinamiğindeki uygulamaları çok azdır.

$\mathrm{Bu}$ durum ise bizi conformable kesirsel mertebeden dinamik sistemlerin popülasyon dinamiğindeki uygulamalarına itmiştir. (11) modeli için parametre değerleri biyolojiden bağımsız alınarak matematiksel olarak doğruluğu araştırılmıştır. Model popülasyon dinamikleri için var olan birçok dinamik davranışı (kararlı durum, kaos) başarılı bir şekilde yansıtmaktadır.

\section{Yazarların Katkısı}

Çalışmaya her iki yazar da eşit oranda katkı sunmuştur.

\section{Çıkar Çatışması Beyanı}

Yazarlar arasında herhangi bir çıkar çatışması bulunmamaktadır.

\section{Araştırma ve Yayın Etiği Beyanı}

Yapılan çalışmada araştırma ve yayın etiğine uyulmuştur.

\section{Kaynaklar}

[1] Abbas S., Banerje M., Momani S. 2011. Dynamical Analysis of Fractional-Order Modified Logistic Model. Compu. Math. Appl., 62: 1098-1104.

[2] Parra G.G., Arenas A.J., Chen-Charpentier B.M. 2014. A Fractional Order epidemic Model for the Simulation of Outbreaks of Influenza A(H1N1). Math. Method. Appl. Sci., 37: 2218-2226.

[3] Khalil R., Al Horani M., Yousef A., Sababheh M. 2014. A new definition of fractional derivative. J. Comput. Appl. Math., 264: 65-70.

[4] Abdeljawad T. 2015. On conformable fractional calculus. J. Comput. Appl. Math., 279: 57-66.

[5] Perez J.E.S., Gomez-Aguilar J.F., Baleanu D., Tchier F. 2018. Chaotic Attractors with Fractional Conformable Derivatives in the Liouville-Caputo Sense and Its Dynamical Behaviors. Entropy, 20: 384.

[6] Balc1 E., Öztürk İ., Kartal Ş. 2019. Dynamical behaviour of fractional order tumor model with Caputo and conformable fractional derivative. Chaos. Soliton. Fract., 123: 43-51. 
[7] Kartal S., Gurcan F. 2019. Discretization of conformable fractional differential equations by a piecewise constant approximation. Int. J. Comput Math., 96: 1849-1860.

[8] Gürcan F., Kaya G., Kartal Ş. 2019. Conformable fractional order Lotka-Volterra predator-prey model: Discretization, stability and bifurcation. J. Comput. Nonlin. Dyn., 14 (11): 111007.

[9] Ye H., Ding Y. 2009. Nonlinear Dynamics and Chaos in a Fractional-Order HIV Model. Math. Probl. Eng., 2009: 1-12.

[10] Ertürk V.S., Odibat Z.M., Momani S. 2011. An Approximate Solution of a Fractional Order Differential Equation Model of Human T-Cell Iymphotropic Virus I (HTLV-I) Infection of CD4+ T-Cells. Comput. Math. Appl., 62: 996-1002.

[11] Zeng C., Yang Q. 2010. A Fractional Order HIV Internal Viral Dynamics Model. Comput. Model. Eng.Sci., 59: 65-77.

[12] Özalp N., Demirci E. 2011. A Fractional Order SEIR Model with Vertical Transmission. Math. Comput. Model., 54: 1-6.

[13] Pinto C.M.A., Machado J.A.T. 2013. Fractional Model for Malaria Transmission Under Control Strategies. Comput. Math. Appl., 66: 908-916.

[14] Ahmed E., Elgazzar A.S. 2007. On Fractional Order Differential Equations Model for Nonlocal Epidemics. Physicia A, 379: 607-614.

[15] Rihan F.A. 2013. Numerical Modelling of Fractional-Order Biological Systems, abstr. Appl. Anal., 2013: 1-11.

[16] Varalta N., Gomes A.V., Camargo R.F. 2014. A Prelude to the Fractional Calculus Applied to Tumor Dynamics. Tendencias em Matematica Aplicade e Computacional., 15: 211-221.

[17] Ahmed E., Hashis A.H., Rihan F.A. 2012. On Fractional Order Cancer Model. J. Fract. calc. Appl., 3: 1-6.

[18] Shanbazi M., Erjaee G.H., Erjaee H. 2014. Dynamical Analysis of chemotherapy Optimal Control Using Mathematical Model Presented by Fractional Differential Equations, Describing Efector Immune and Cancer Cells Interactions. Journal of Pharmacy and Pharmaceutical Sciences, 3: 517.

[19] Bozkurt F. 2014. Stability Anaysis of a Fractional Order Differential equation System of a GBMIS Interaction Depending on the Density. Appl. Math. Inf. Sci., 8: 1021-1028.

[20] Jun D., Jun Z.G., Yong X., Hong Y., Jue W. 2014. Dynamic Behavior Analysis of FractionalOrder Hindmarsh-Rose Neuronal Model. Cong. Neurodyn., 8: 167-175.

[21] El-Raheem Z.F., Salman S.M. 2014. On a Discretization Process of Fractional-Order Logistic Differential Equation. J. Egyptian. Math. Soc., 22: 407-412.

[22] Arafa A.A.M., Rida S.Z., Khalil M. 2013. The Effect of Anti-Viral drug Treatment of Human Immunodeficiencey Virus Type 1 (HIV-1) Described by a Fractional Order Model. Appl. Math. Model., 37: 2189-2196.

[23] Arafa A.A.M., Rida S.Z., Khalil M. 2014. A Fractional-Order Model of HIV Infection with Drug Therapy Effect. J. Egyptian Math. Soc., 22: 538-543.

[24] Yan Y., Kou C. 2012. Stability Analysis for a Fractional Differential Model of HIV Infection of CD4+ T-Cells with Time Delay. Math. Comput. Simulat., 82: 1572-1585.

[25] Gökdoğan A., Yıldırım A. 2011. Merdan, M., Solving a Fractional Order Model of HIV Infection of CD4+ T-Cells. Math. Cumput. Model., 54: 2182-2138.

[26] Matouk A.E. 2009. Stability Conditions, Hyperchaos and Control in a Novel Fractional Order Hyperchaotic System. Phys. Lett. A., 373: 2166-2173.

[27] Jafari H., Daftardar-Gejji V. 2006. Solving a System of Nonlinear Fractional Differential equations Using Adomian Decomposition. J. Math. Anal. Appl., 196: 644-651.

[28] Odibat Z., Momani S. 2008. Numerical Methods for Nonlinear Partial Differential Equations of Fractional Order. Appl. Math. Modelling., 32 (1): 28-39.

[29] Ajou A.E., Odibat Z., Momani S., Alawneh A. 2010. Construction of Analytical solutions to Fractional Differential Equations Using Homotopy Analysis Method. IAENG Int. J. Appl. Math., 40 (2).

[30] Ünlü C., Jafari H., Baleanu D. 2013. Revised Variational Iteration Method for Solving systems of Nonlinear fractional Order differential Equations. Bstr. Appl. Anal., 2013: 1-7.

[31] Amen I., Novati P. 2017. The Solution of Fractional Order epidemic Model by Implicit Adams Methods. Aplied Mathematical Modelling, 43: 78-84. 
[32] Ertürk V.S., Momani S. 2008. Solving systems of Fractional Differential Equations Using differential Transform Method. J. Comput. Appl. Math., 215: 142-151.

[33] Verhulst P.F. 1838. A note on the law of population growth. Correspondence Mathema- tique et Physique Publee par A. Quetelet, 10: 113-121.

[34] Zhou S., Zhang W., He Z. 2009. Generalized projective synchronization of time-delayed fractional order chaotic systems. International Conference on Communications, Circuits and Systems, Milpitas, CA, 853-857.

[35] Wang D., Yu J. 2008. Chaos in the fractional order logistic delay system. International Conference on Communications, Circuits and Systems, Fujian, 646-651.

[36] El-Saka H.A.A., Ahmed E., Shehata M.I., El-Sayed A.M.A. 2008. On stability, persistence, and Hopf bifurcation in fractional order dynamical systems. Nonlinear Dynamics, 56 (1): 121-126.

[37] Elaydi S. 2005. An Introduction to Difference Equations. Springer, 1-539. 